\title{
How common are vascular injuries in open tibial fractures? A prospective longitudinal cohort study
}

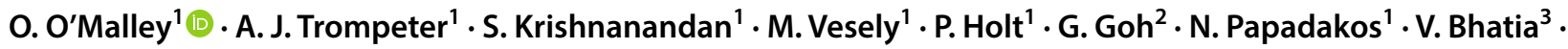 \\ C. B. Hing ${ }^{1}$
}

Received: 21 November 2018 / Accepted: 5 March 2019 / Published online: 13 March 2019

(c) The Author(s) 2019

\begin{abstract}
Background Tibial fractures have an incidence of $15 \%$ of all adult fractures. They have been shown to have the highest incidence of non-union in long bone fractures and the highest incidence of vascular injury. Evidence from the literature suggests that a good vascular supply is important to ensure bone union. The aim of our study was to prospectively assess the incidence of vascular injuries in open tibial fractures and determine whether they were associated with an increased risk of non-union. Methods We performed a prospective study to investigate the incidence of arterial injuries with computed tomography angiography (CTA) in patients with Gustilo-Anderson grade I-III open tibial fractures between 2013 and 2015. CTA was performed with the trauma series at acute admission and reported by two independent musculoskeletal radiologists. Patients were followed up with clinical and radiographic assessment for 1 year.

Results We recruited 77 patients into the study, and 56 patients ( 47 males, 9 females) were available for the final analysis, between 16 and 90 years of age. At the initial assessment, 29\% had signs of arterial injury with active extravasation in 5\%. The most common site of injury was in the diaphysis (87.5\%), and the commonest mechanism was a road traffic accident. We found no significant relation between occult vascular injury and non-union $(p>0.05)$.

Conclusion The incidence of vascular injury in open tibial fractures is $29 \%$, and CTA is therefore a useful test in identifying vascular injuries that may require vascular intervention.
\end{abstract}

Keywords Tibia $\cdot$ Angiogram $\cdot$ Vascular injury

\section{Introduction}

Tibial shaft fractures have an incidence of $15 \%$ of all adult fractures and are high-energy injuries often associated with significant soft tissue injury [1]. The main aims of treatment are to restore alignment of the tibia with adequate stability to allow bone union and rehabilitation of the limb. In the United Kingdom (UK), treatment follows the British Orthopaedic Association and British Association of Plastic, Reconstructive and Aesthetic Surgeons guidelines of a

O. O’Malley

oliviaomalley@doctors.org.uk

1 Department of Trauma and Orthopaedics, St George's University Hospitals NHS Foundation Trust, London, UK

2 Department of Radiology, The Alfred Hospital, Melbourne, Australia

3 St George's University London, St George's University Hospitals NHS Foundation Trust, London, UK combined orthoplastic approach to management in regional specialist centres $[2,3]$.

Evidence from the literature suggests that a good vascular supply is important to ensure bone union with vascular compromise being implicated in non-union [4-7]. Tibial fractures have been shown to have the highest incidence of non-union of all long bone fractures as well as the highest incidence of vascular injury [8-10]. The incidence of vascular injury associated with type III tibial fractures has been quoted as 9\% [11].

Diagnosis of vascular injury at the initial assessment relies on clinical examination with palpable pulses inferring intact vascular supply. However, in the absence of obvious signs of vascular compromise and critical limb ischaemia, vascular injuries may be easily missed yet controversy exists as to whether routine investigation or exploration of open tibial fractures with palpable pulses is justified [12, 13]. It may be difficult to appreciate the degree of internal soft tissue injury on the initial inspection of wound size 
and imaging, including the presence or absence of vascular injury. However, a recent systematic review of the literature has shown that computed tomography angiogram (CTA) should be the investigation of choice in patients with suspected vascular injury in any anatomic region [14]. CTA is the examination of choice due to its short acquisition time and high diagnostic accuracy. Studies have shown the specificity and sensitivity to be $98.7-100 \%$ and $90-95.2 \%$, respectively, for CTA diagnosis of arterial damage in the extremities following trauma. Signs eliciting vascular injury include active extravasation of contrast material, incidence of pseudoaneurysm formation, abrupt narrowing of an artery, loss of opacification of a segment of an artery and arteriovenous fistula formation $[15,16]$.

The aim of this study was to prospectively elucidate the incidence of vascular injuries associated with open tibial fractures and determine whether vascular injury correlated to an increased risk of non-union.

\section{Methods}

We conducted a prospective longitudinal cohort observational study to assess the incidence of vascular injury in a consecutive series of patients admitted to a level 1 trauma centre in the United Kingdom (UK) with open tibia fractures between April 2013 and November 2015. All patients admitted directly or as part of a tertiary referral pathway were invited to participate in the study. Ethics approval was obtained from the local Research Ethics Committee (IRAS 95683, REC 12/LO/1646). Patients were invited to participate if they had an open fracture of the tibial shaft, plateau or pilon, were greater than 16 years of age and could give informed consent (Table 1). Patients were excluded if they were unable to give consent or follow the study protocol, had a previous vascular injury or had an ipsilateral limb injury including a vascular injury.

All patients enrolled into the study were managed as per the standard British Orthopaedic Association and British Association of Plastic, Reconstructive and Aesthetic Surgeons (BOAST/BAPRAS 4 Algorithm) guidelines from 2013 [2]. The patients received joint care from an orthopaedic and plastic surgeon, and surgical wound debridement and

Table 1 Inclusion and exclusion criteria

\begin{tabular}{ll}
\hline Inclusion criteria & Exclusion criteria \\
\hline Open tibial fractures & $\begin{array}{c}\text { Ipsilateral limb injury } \\
\text { with vascular injury }\end{array}$ \\
$>16$ years & Previous vascular injury \\
Conscious or unconscious & Ankle fractures \\
Single limb or polytrauma & Unable to consent \\
\hline
\end{tabular}

operative fracture stabilisation were performed within $24 \mathrm{~h}$ and definitive soft tissue cover within $72 \mathrm{~h}$.

At the initial assessment, general demographics (age, gender and smoking) were recorded together with the injury mechanism, fracture classification, soft tissue injury classification (Gustilo and Anderson [17]) and details of the initial surgical management were recorded.

All patients recruited into the study received a CTA at the time of a trauma series CT scan in the emergency department (ED). This was done within $60 \mathrm{~min}$ as per NHS England guidance [18]. At $4 \mathrm{ml} / \mathrm{s}, 90 \mathrm{ml}$ Omnipaque 300 was used as the contrast medium, and the scan was performed from L2 to the feet in arterial phase. The CTA required an additional $3.6 \mathrm{mSv}$ of radiation exposure in addition to the trauma series CT. Two independent radiologists reviewed each CTA.

All patients received standard routine postoperative management including physical therapy, occupational therapy and psychological support as per their injury, their means of skeletal fixation and their associated injuries.

The primary outcome was the incidence of vascular injury as reported by CTA. Secondary outcomes included non-union in subjects with open fractures and concomitant vascular injury noted on CTA. Time to union was defined by the radiographic union score in the tibia (RUST) score at 6 months [19]. All patients were independently reviewed in a research clinic at 2 weeks, 6 weeks, 3 months, 6 months and 1 year from injury using the SF-36 and visual analogue scale for pain (VAS) scores [20, 21].

Data were collected, anonymised and entered into a standardised spreadsheet (MS Excel, Microsoft, Washington, USA). Basic demographic data were recorded, and incidence of vascular injury was calculated. The association between vascular injury and non-union was analysed with chi-squared testing using SPSS statistics package (IBM Corp. Released 2013. IBM SPSS Statistics for Windows, version 21.0. Armonk, NY).

A significant difference was defined by $p<0.05$.

\section{Results}

Between 2013 and 2015, 78 patients were recruited into the study and after exclusions and withdrawals, the total number of patients available for the final analysis was 56 (Table 2). All patients included in the study had palpable pulses at admission. All patients had the initial debridement within $24 \mathrm{~h}$ and had soft tissue cover within $72 \mathrm{~h}$ as per BOAST guidelines.

The incidence of vascular injury on CTA was $29 \%$ (16 patients). A summary of the patients with vascular injury on CTA is found in Table 3. Those with abnormal CTA had a variety of fracture patterns, but a predominance 
Table 2 Patient demographics

\begin{tabular}{|c|c|}
\hline \multicolumn{2}{|l|}{ Age (years) } \\
\hline Range & $16-90$ \\
\hline Mean & 41 \\
\hline \multicolumn{2}{|l|}{ Gender } \\
\hline Male (\%) & $47(84 \%)$ \\
\hline Female $(\%)$ & $9(16 \%)$ \\
\hline \multicolumn{2}{|l|}{ Mechanism of injury } \\
\hline Road traffic accident (RTA) & 31 \\
\hline Fall & 18 \\
\hline Other (sports etc.) & 5 \\
\hline Not specified & 2 \\
\hline \multicolumn{2}{|l|}{ Length of hospital stay (days) } \\
\hline Range & $3-63$ \\
\hline Mean & 15 \\
\hline \multicolumn{2}{|l|}{ AO classification } \\
\hline 42-A1 & 8 \\
\hline $42-\mathrm{A} 2$ & 7 \\
\hline $42-\mathrm{A} 3$ & 4 \\
\hline $42-\mathrm{B} 1$ & 2 \\
\hline $42-\mathrm{B} 2$ & 5 \\
\hline $42-\mathrm{B} 3$ & 5 \\
\hline $42-\mathrm{C} 2$ & 8 \\
\hline $42-\mathrm{C} 3$ & 9 \\
\hline 43-A1 & 1 \\
\hline $43-\mathrm{A} 3$ & 2 \\
\hline 43-B1 & 1 \\
\hline $43-B 2$ & 1 \\
\hline $43-\mathrm{C} 2$ & 1 \\
\hline $43-\mathrm{C} 3$ & 2 \\
\hline \multicolumn{2}{|l|}{ Gustilo-Anderson grade } \\
\hline $3 \mathrm{a}$ & $23(41.1 \%)$ \\
\hline $3 b$ & $17(30.3 \%)$ \\
\hline $3 \mathrm{c}$ & $16(28.6 \%)$ \\
\hline
\end{tabular}

for the diaphyseal region $(87.5 \%)$ and the predominant mechanism of injury was a high-velocity incident.

The anterior tibial artery (ATA) was the most commonly affected artery on CTA reported in 11 out of 16 cases of vascular injury (69\%) (Fig. 1). The most common finding being the fracture displacing bone lying in close association to the artery results in attenuated flow. Two ATA injuries involved active extravasation. The peroneal artery was affected in four cases with one case of active extravasation. The posterior tibial artery was injured in three cases and the dorsalis pedis in one case. In all cases of extravasation, road traffic accident (RTA) was the mechanism of injury.

\section{Bone union}

There were four patients (seven percentage) who had nonunion and required further surgery as shown in Table 4. Two of these patients did not have a vascular injury and two had a vascular injury. Both patients who had a vascular injury had injuries to the anterior tibial artery. All of the original injuries that led to non-union were in the diaphysis with 75\% being multi-fragmentary fractures. With a chi-squared statistic of 1.12, there was no relation between vascular injury and non-union.

\section{Outcome scores}

The mean VAS at 6 weeks was 3, at 3 months was 4.2 , at 6 months was 3.9 and at 1 year was 3.4. Pain scores generally decreased overtime as would be expected; however, a lower mean pain score was recorded at the initial followup at 6 weeks. In those with vascular injuries, mean VAS scores were higher at each time interval 4.3, 5.9, 4.5 and 4.6, respectively. This indicates higher pain scores in those with a vascular injury, and this could be attributed to severity of their injury.

SF-36 was used as a measure of health-related quality of life and again was recorded at 6 weeks, 3 months, 6 months and 1 year with 91, 93, 95 and 102 as the mean results, respectively. This showed that patients felt their quality of life improved as time progressed as to be expected. In those with vascular injury, mean scores were 89, 96, 88 and 104.5, respectively. This showed a general increase in quality of life with an anomaly at 6 months. Quality of life was lower in those in vascular injury at 5 weeks and 6 months compared to those without, but was higher after 1 year.

\section{Discussion}

We found the incidence of vascular injury in open tibial fractures is to be $29 \%$. This study is the first to prospectively assess the incidence of vascular injury with CTA in lower limb open fractures. Previous literature on concomitant vascular injury in open tibial fractures does not assess incidence directly but rather outcomes of the fracture [9].

The incidence of vascular injury in our study was higher than those previously described; however, only three cases involved 5\% active extravasation $[5,11]$. This may relate to a small vessel, which has no clinical significance on healing and recovery as demonstrated by these causes going on to uncomplicated union. Furthermore, many of the vascular injuries described were reversible when fracture had been reduced, and therefore, if the CTA was preformed postfracture stabilisation the incidence of vascular injury may have been lower. 
Table 3 Abnormal CTA patients

\begin{tabular}{|c|c|c|c|c|c|}
\hline Age (years) & $\mathrm{AO}$ & Injury & Artery injured & Type of injury & Union \\
\hline 38 & $42-\mathrm{C} 2$ & Hit by train & $\mathrm{ATA}^{\mathrm{b}}$ & Occlusion at fracture level & Non-union \\
\hline 90 & $43-\mathrm{B} 2$ & Fall & ATA and $\mathrm{PTA}^{\mathrm{c}}$ & Occluded proximally athersclerosis & Deceased \\
\hline 30 & $42-\mathrm{C} 2$ & $\mathrm{RTA}^{\mathrm{a}}$ & PTA & Vessel contusion/spasm & Union \\
\hline 51 & $42-\mathrm{C} 2$ & Not given & ATA & $\begin{array}{l}\text { Filling defect distal to fracture, complete effacement at distal fracture } \\
\text { line }\end{array}$ & Non-union \\
\hline 41 & 42-A1 & Fall from horse & ATA & Not visible distal to fibular fracture & Union \\
\hline 52 & $42-\mathrm{A} 2$ & RTA & ATA and peroneal & Opacify for segment at open fracture flow reconstitutes distally & Union \\
\hline 53 & $42-\mathrm{A} 2$ & RTA & ATA & Spasm & Union \\
\hline 20 & $42-\mathrm{B} 2$ & RTA & Peroneal & Loss of opacification at fracture level with extravasation & Union \\
\hline 41 & $42-\mathrm{B} 2$ & RTA & ATA & Loss of opacification at fracture level & Union \\
\hline 36 & $42-\mathrm{C} 3$ & RTA & $\begin{array}{l}\text { Dorsalis pedis } \\
\text { and common } \\
\text { peroneal }\end{array}$ & Do not opacify below fracture & Union \\
\hline 32 & $42-C 2$ & Fall & ATA & Loss of opacification distal to fracture possible due to spasm & Union \\
\hline 26 & $42-\mathrm{C} 3$ & RTA & ATA & Active extravasation and haematoma & Union \\
\hline 24 & 42-A1 & RTA & PTA & Occlusion & Union \\
\hline 17 & $43-\mathrm{C} 3$ & RTA & ATA & No opacification distal to fracture possible spasm & Union \\
\hline 40 & $42-\mathrm{A} 1$ & RTA & ATA & Extravasation at level of fracture & Union \\
\hline 50 & $42-B 3$ & RTA & Peroneal & Non-specified injury & Union \\
\hline
\end{tabular}

${ }^{a} R T A$ road traffic accident

${ }^{\mathrm{b}} A T A$ anterior tibial artery

${ }^{\mathrm{c}} P T A$ posterior tibial artery

Fig. 1 Radiograph of an open tibial fracture and CTA showing associated vascular injury to anterior tibial artery
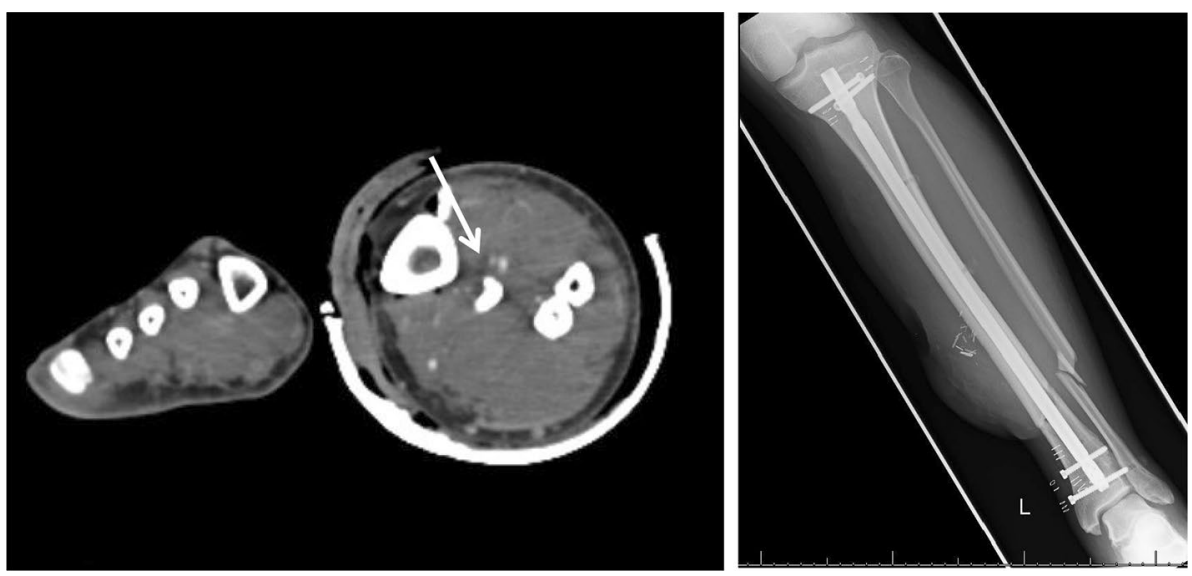

Table 4 Cases of non-union

\begin{tabular}{lllll}
\hline Age (years) & Mechanism & Gustilo & Anderson & $\begin{array}{l}\text { Vas- } \\
\text { cular } \\
\text { injury }\end{array}$ \\
\hline 38 & Train & $3 \mathrm{c}$ & $42-\mathrm{C} 2$ & $\mathrm{Y}^{\mathrm{b}}$ \\
51 & Unknown & $3 \mathrm{c}$ & $42-\mathrm{C} 2$ & $\mathrm{Y}$ \\
54 & RTA $^{\mathrm{a}}$ & $3 \mathrm{~b}$ & $42-\mathrm{C} 3$ & $\mathrm{~N}^{\mathrm{c}}$ \\
64 & Fall & $3 \mathrm{~b}$ & $42-\mathrm{A} 1$ & $\mathrm{~N}$ \\
\hline
\end{tabular}

${ }^{a} R T A$ road traffic accident

${ }^{\mathrm{b}} Y$ yes

${ }^{\mathrm{c}} N$ no
Palpable pulses were felt in all patients on admission; however, 29\% were discovered to have vascular injury. Although immediate vascular intervention was not required, the findings would alert physicians to potential need for intervention and ongoing surgical planning.

In terms of the pattern of vascular injury found, the anterior tibial artery was most commonly affected which is similar to previous literature [5, 22-24]. This could be assumed as a result of anterior force commonly resulting from an RTA. We found high-impact injuries such as RTA to be the most common cause of vascular injury, which agrees with previous literature [8]. 
CTA has previously been proposed as the investigation of choice for suspected arterial injury in trauma. It is quick and readily available, with relatively few contraindications or complications. We had no adverse effects or extra morbidity from the additional angiography sequence in our series, and this correlates with other studies [16]. The vast majority of trauma patients have a CT head to pelvis, and therefore, angiography may be suitable with little extra ionising radiation or risk.

A previous study concluded that routine use of CTA in lower extremity fracture was not indicated. However, the presence of open fracture, distal or shaft tibial fractures increases the risk of having vascular injury on CTA. They also concluded that all their patients who had diminished or absent pulses required vascular treatment and therefore emphasise how important clinical examination is [16].

The literature explains how early exploration and appropriate surgery may lessen the need for amputation in those with vascular injury. The exact nature of the vascular injuries must be detected promptly to minimise the length of ischaemia and revascularisation should be carried out whenever it is possible [13]. The type of vascular injury can also predict the risk of reconstructive complications [24]. Therefore, early recognition of a vascular injury using CTA can aid prompt surgical management and follow-up planning.

In terms of our secondary outcome, non-union in vascular injury, we found no association. This contradicts a previous study that found those with vascular occlusion had a significantly greater incidence of delayed union or non-union [6].

A previous study found a significant relationship between posterior tibial artery injury and non-union; none of the cases identified in our study with posterior tibial artery injury had non-union. Of our non-unions, all were in the diaphysis and $75 \%$ were multi-fragmentory, which fits with the results of a previous study [9].

The literature is limited in terms of incidence of vascular injury in open tibial fractures and its direct association with non-union. Although this is the first study to look prospectively at vascular injury in open fractures, it would be useful to gain a larger sample of those with vascular injuries to further assess their outcomes in terms of union. Retrospective studies with larger sample sizes showed poorer outcomes and statistically significant rate of non-union in those with vascular injury $[4,5]$.

In conclusion, CTA is useful in detecting potential vascular injury; although many may not require vascular intervention, it alerts physicians to those who may require it. CTA should be considered in all those with high-velocity injuries resulting in an open tibial fracture. It is also valuable in preoperative planning for soft tissue reconstructive surgery in order to identify suitable vessels for local or free flaps.
Acknowledgements This study was funded by the AO Foundation, UK.

\section{Compliance with ethical standards}

Conflict of interest The authors declare that they have no conflict of interest.

Open Access This article is distributed under the terms of the Creative Commons Attribution 4.0 International License (http://creativeco mmons.org/licenses/by/4.0/), which permits unrestricted use, distribution, and reproduction in any medium, provided you give appropriate credit to the original author(s) and the source, provide a link to the Creative Commons license, and indicate if changes were made.

\section{References}

1. Aslan A, Uysal E, Özmeriç A (2014) A staged surgical treatment outcome of type 3 open tibial fractures. ISRN Orthop 2014:721041. https://doi.org/10.1155/2014/721041.8

2. Nanchahal J, Nayagam S, Khan U, Moran C, Barrett S, Sanderson F, Pallister I (2009) Standards for the management of open fractures of the lower limb. British Association of Plastic, Reconstructive and Aesthetic Surgeons, London

3. Wordsworth M, Lawton G, Nathwani D et al (2016) Improving the care of patients with severe open fractures of the tibia: the effect of the introduction of Major Trauma Networks and national guidelines. Bone Joint J 98B(3):420-424

4. O'Halloran K, Coale M, Costales T, Zerhusen T et al (2016) Will my tibial fracture heal? Predicting non-union at the time of definitive fixation based on commonly available variables. Clin Orthop Rel Res 474(6):1385-1395

5. Chummun S, Wigglesworth T, Young K et al (2013) Does vascular injury affect the outcome of open tibial fractures. Plast Reconstr Surg 131(2):303-309

6. Dickson K, Katzman S, Delgado E et al (1994) Dealyed unions of open tibial fractures. Correlation with arteriography results. Clin Orthop Rel Res 302:189-193

7. Dickson K, Katzman S, Paiement G (1995) The importance of the blood supply in the healing of tibial fractures. Contemp Orthop 30(6):489-493

8. Howard P, Makin GS (1990) Lower limb fractures with associated vascular injury. J Bone Joint Surg 72(1):116-120

9. Brinker M, Bailey D (1997) Fracture healing in tbial fractures with an associated vascular injury. J Trauma 42(1):11-19

10. Howard M, Court-Brown CM (1997) Epidemiology and management of open fractures of the lower limb. Br J Hosp Med 57(11):582-587

11. Caudle RJ, Stern PJ (1987) Severe open fractures of the tibia. J Bone Joint Surg Am 69(6):801-807

12. Halvorson J, Anz A, Langfitt $M$ et al (2011) Vascular injury associated with extremity trauma: initial diagnosis and management. J Am Acad Orthop Surg 19(8):495-504

13. Waikakul S, Sakkarnkosol S, Vanadurongwan V (1998) Vascular injuries in compound fractures of the leg with initially adequate circulation. J Bone Joint Surg Br 80(2):254-258

14. Patterson B, Holt PJ, Cleanthis M (2012) Imaging vascular trauma. Br J Surg 99(4):494-505

15. Miller-Thomas MM, West OC, Cohen AM (2005) Diagnosing traumatic arterial injury in the extremities with CT angiography: pearls and pitfalls. Radio Graph 25:S133-S142 
16. Monazzam S et al (2017) When are CT angiograms indicated for patients with lower extremity fractures? A review of 275 extremities. J Trauma Acute Care Surg 82:133

17. Gustilo R, Mendoza RM, Williams DN (1984) Probelms in the management of type III (severe) open fractures: a new classification of type III open fractures. J Trauma 24(8):742-746

18. England NHS (2013) NHS standard contract for major trauma service, schedule 2-the services A. Service specification. NHS England, London

19. Litrenta J, Tornetta P, Mehta S et al (2015) Determination of radiographic healing: an assessment of consistency using RUST and modifying RUST in metadiaphyseal fractures. J Orthop Trauma 29(11):516-520

20. Ware J, Sherbourne CD (1992) The MOS 36-item short-form health survey (SF-36). Conceptual framework and item selection. Med Care 30(6):473-483

21. Burckhardt C, Jones KD (2003) Adult measures of pain: the McGill pain questionnaire (MPQ), rheumatoid arthritis pain scale (RAPS), short-form McGill pain questionnaire (SF-MPQ), verbal descriptive scale (NDS), visual analogue scale (VAS), and
Western Haven-Yale multidisciplinary pain Arthritis Rheum. Arthritis Care Res 49:S96-S104

22. Haddock NT, Weichman KE, Reformat DD, Kligman BE, Levine JP, Saadeh PB (2010) Lower extremity arterial injury patterns and reconstructive outcomes in patients with severe lower extremity trauma: a 26-year review. J Am Coll Surg 210(1):66-72

23. Chen HC, Chuang CC, Chen S, Hsu WM, Wei FC (1994) Selection of recipient vessels for free flaps to the distal leg and foot following trauma. Microsurgery 15:358-363

24. Stranix J, Lee ZH, Jacoby A, Anzai L, Avraham T, Thanik V, Saadeh P, Levine J (2017) Not all gustilo type IIIB fractures are created equal: arterial injury impacts limb salvage outcomes. Plast Reconstr Surg 140(5):1033-1041

Publisher's Note Springer Nature remains neutral with regard to jurisdictional claims in published maps and institutional affiliations. 